\title{
Asymptomatic Case of Cervical Vagal Schwannoma
}

\begin{abstract}
Schwannomas are benign, slow-growing, and encapsulated tumors deriving from the perineural cells located in the nerve sheath. They can arise from any peripheral, cranial, or autonomic nerves and show a predilection for the head and neck region. Extracranial head and neck schwannomas are rare tumors. They may produce secondary symptoms such as nasal obstruction, dysphagia, and hoarseness of voice depending on the location of the tumor. The preoperative diagnosis is difficult. Although preoperative imaging or fine needle aspiration cytology may help to reveal diagnosis, they are inadequate. The definitive diagnosis is made by histopathological examinations.
\end{abstract}

Keywords: Benign tumor, encapsulated tumor, hoarseness of voice, schwannoma

\section{Introduction}

Cervical vagal schwannomas are rare, slow-growing tumors usually reported to occur in patients between 30 and 50 years of age. Males and females are equally affected. They are usually asymptomatic benign lesions, and complete surgical resection is the treatment of choice. Imaging plays a central role in diagnosing and in optimal surgical treatment in vagal nerve neoplasm.

\section{Case Report}

A 28-year-old female was admitted to our department for swelling on the left side of the neck. On palpation, a soft, smooth-surfaced mass on the left cervical region (at Level II), measuring $5 \mathrm{~cm} \times 3 \mathrm{~cm}$, was present. Neither paroxysmal cough nor bradycardia on palpation was observed. Cranial nerve examination was normal.

Ultrasound (US) of the neck showed a well-defined heterogeneous lesion of size $4.5 \mathrm{~cm} \times 2.6 \mathrm{~cm}$ with necrotic areas within left postauricular and infra-auricular region, showing minimal vascularity on Doppler imaging. Fat plane with adjacent muscles and vessels is maintained. Computed tomography (CT) scan of the neck demonstrated a well-defined heterogeneously enhancing hypodense lesion of approximate

This is an open access journal, and articles are distributed under the terms of the Creative Commons Attribution-Non Commercial-ShareAlike 4.0 License, which allows others to remix, tweak, and build upon the work non-commercially, as long as appropriate credit is given and the new creations are licensed under the identical terms.

For reprints contact: reprints@medknow.com size $5.3 \mathrm{~cm} \times 4.2 \mathrm{~cm} \times 3.2 \mathrm{~cm}$ with nonenhancing necrotic areas within deep to the left sternocleidomastoid muscle extending from $\mathrm{C} 2$ to $\mathrm{C} 6$ vertebral level. The lesion is compressing and displacing internal jugular vein anterolaterally abutting left internal carotid artery, posterior belly of digastric muscle anteriorly, and splenius and semispinalis muscles posteromedially. The fat plane between lesion and adjacent soft tissue is maintained [Figures 1 and 2].

The results of fine needle aspiration cytology of the mass, performed before the admission, were inconclusive. The patient, therefore, underwent surgery. Under general anesthesia, a cervical incision along the anterior border of the sternocleidomastoid muscle was made and the dissection proceeded beneath the muscle. A grayish-white, round-shaped mass was observed, measuring $\sim 3 \mathrm{~cm} \times 3 \mathrm{~cm}$ lying between the left carotid artery and the internal jugular vein. Both the superior and inferior ends of the mass appeared in continuity with the vagus nerve. Multiple tiny nerve branches were seen around the tumor [Figure 3].

The tumor was removed leaving the capsule of the tumor, preserving the tiny branches of the vagus [Figure 4].

\section{Surgical technique}

The nerve-sparing subcapsular resection technique for schwannomas is based on the principle that nerve fibers get expanded and stretched out on the tumor rather than

How to cite this article: Sonone JK, Nagpure PS, Puttewar MP, Chavan S, Garg D. Asymptomatic case of cervical vagal schwannoma. Indian J Med Paediatr Oncol 2018;39:391-4.

\section{Jayant Krishna Sonone, Prakash S Nagpure, Manish P Puttewar, Sushil Chavan, Deepika Garg}

Department of ENT, MGIMS, Sevagram, Maharashtra, India

Address for correspondence: Dr. Jayant Krishna Sonone, Department of ENT, MGIMS, Sevagram, Wardha - 442 102, Maharashtra, India.

E-mail: jayantsonone@hotmail. com

Access this article online Website: www.ijmpo.org

DOI: 10.4103/ijmpo.ijmpo_225_17 Quick Response Code:

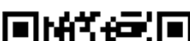




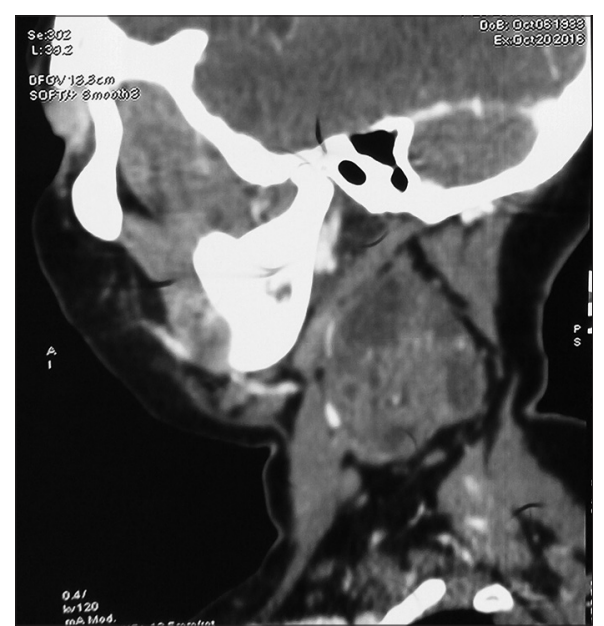

Figure 1: Contrast-enhanced computed tomography neck (sagittal section) showing mass occupying posterior triangle

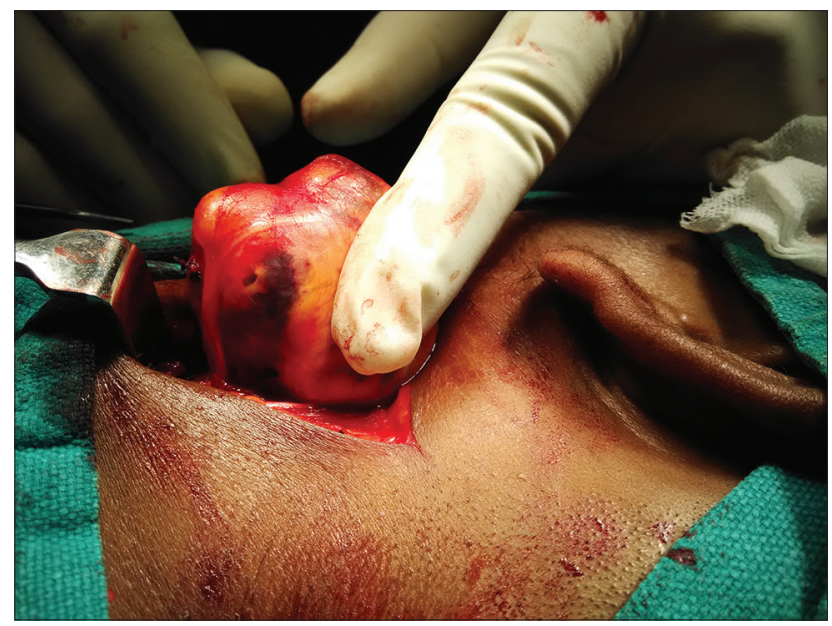

Figure 3: Intraoperative photograph showing vagal schwannoma

getting embedded within it. The technique described herein potentially preserves these nerve fibers without significantly compromising nerve function as nerve continuity is maintained.

After identifying the location of the important blood vessels and nerves in relation to the tumor, the mass is delineated on all sides. The proximal and distal ends of the nerve abutting the tumor are identified, and meticulous sharp dissection is carried out, parallel to the axis of the nerve (minimizing damage to collateral nerve fibers), until the cleavage plane between the tumor tissue and tumor capsule is reached. Once in the subcapsular plane, the tumor mass is carefully freed proximally, distally, and from the sides, and then excised. Care is taken to minimize traction to the surrounding nerve fibers. The tumor is thus resected with preservation of the majority of nerve fibers.

The histopathological examination confirmed the diagnosis of benign schwannoma of the vagus nerve. Postoperatively, the patient does not develop any hoarseness of voice, and examination of the larynx was normal. At follow-up,

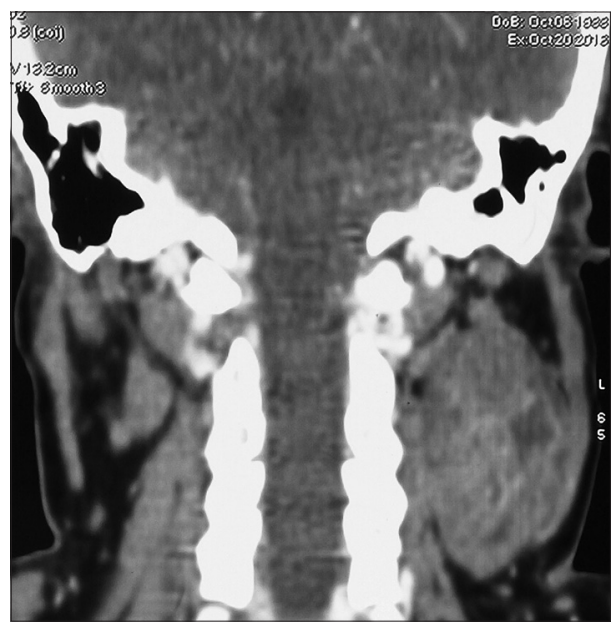

Figure 2: Contrast-enhanced computed tomography neck (coronal section) showing mass occupying posterior triangle

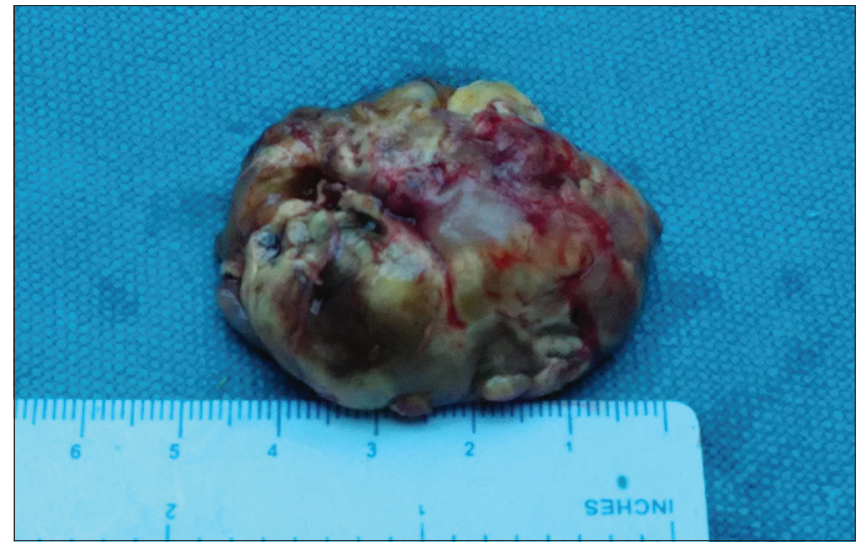

Figure 4: Specimen of the left cervical vagal schwannoma

15 days after surgery, the patient was well, without evidence of disease and with no vocal cord palsy.

\section{Discussion}

Schwannomas are rare peripheral nerve tumours; about one third occur in the head and neck region. ${ }^{[1]}$ Clinically, they present as asymptomatic slow-growing lateral neck masses that can be palpated along the medial border of the sternocleidomastoid muscle.

Pre-operative diagnosis of schwannoma is difficult because many vagal schwannomas do not present with neurological deficits and several differential diagnoses for tumour of the neck may be considered, including paraganglioma, branchial cleft cyst, malignant lymphoma, metastatic cervical lymphadenopathy. ${ }^{[2]}$ As schwannomas are mostly asymptomatic we should have an index of suspicion for this tumour.

Magnetic Resonance Imaging (MRI) is the investigation of choice. In this case patient has undergone Computed Tomography (CT) of neck. We reached the definitive diagnosis by histopathological examination showing 


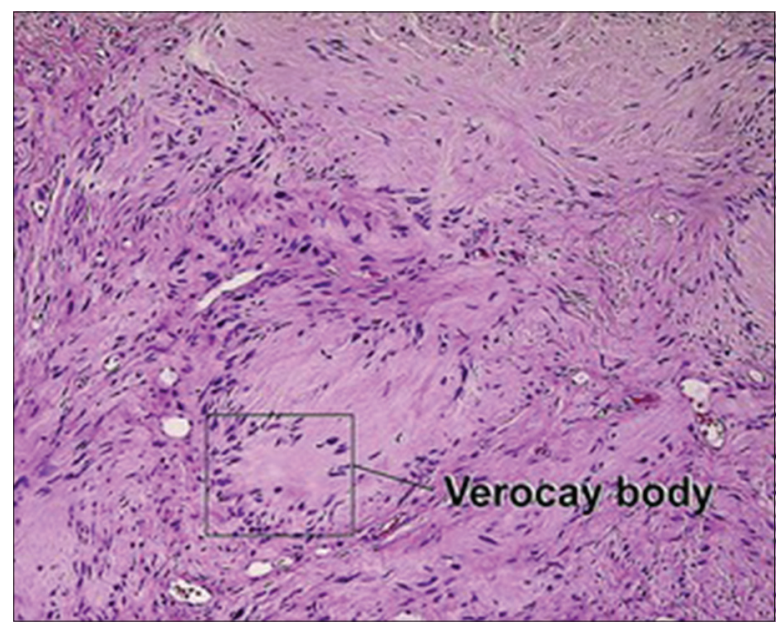

Figure 5: Verocay body with palisaded rows around it

presence of Verocay bodies which are definitive for schwannoma [Figure 5].

Treatment of vagal nerve tumours is complete surgical excision. At surgery, these tumours appear as yellowishwhite, well-circumscribed masses. Dissection of the tumour from the vagus with preservation of the neural pathway should be the primary aim of surgical treatment for these tumours. Incomplete treatment, such as open biopsy, should be avoided, since it makes definitive excision of the tumour much more difficult.

As schwannomas are benign, all efforts should be made to preserve nerve function so that quality of life is unaffected. Valentino et al. ${ }^{[3]}$ considered neural deficits to be the result of tumour neuritis, and Russel and Rubinstein ${ }^{[4]}$ reported neural fibres within schwannomas to be the cause of such deficits, independent of the employed surgical technique. Various techniques have been described for removal of these tumours, including: tumour excision with neural sacrifice followed by primary anastomosis or neural graft interposition; tumour excision with neural preservation; tumour enucleation between adjacent healthy nerve fibres when possible; tumour emptying (preserving the tumour capsule); and even the 'shelling out' of the tumour (leaving gross tumour inside the capsule). The nerve-sparing subcapsular resection technique for schwannomas described in the current study potentially preserves the nerve fibres, without significantly compromising the nerve function as nerve continuity is maintained.

A literature review by Valentino et al. showed that the various surgical techniques offered different function preservation rates ${ }^{[3]}$ In those who underwent tumour excision without neural sacrifice, 64 per cent had permanent neural deficits, while 29 per cent had temporary deficits. Patients who had their tumours either enucleated or shelled out had a permanent neural deficit rate of 29 per cent and a transient deficit rate of 42 per cent. In those who underwent a shelling out procedure, 29 per cent had no neural deficits at all.
The reported incidence of pre-operative vocal cord paralysis is about $12 \%$, but hoarseness is almost always present following surgery. Therefore, pre-operative assessment of vocal cord mobility should be strongly recommended. Although it is very rare, clinicians should bear in mind the possibility of a nerve sheath tumour in the presence of a neck mass. Pre-operative suspicion is very important, because the patient, and the patient's family, should be informed about the possible post-operative neurological complications, ${ }^{[5]}$ as far as postoperative vocal cord palsy is concerned, an incidence of $85 \%$ has been reported. ${ }^{[6,7]}$ Furthermore, since vagal schwannomas are almost invariably benign in nature, a conservative approach should always be considered in first instance. ${ }^{[2,8]}$ In the presence of post-operative vocal cord palsy, aggressive voice therapy, for vocal cord compensation, should be started soon after surgery.

\section{Conclusion}

Cervical schwannomas, which most often present as asymptomatic unilateral neck masses, are rare tumors. The preoperative diagnosis may be difficult, and it is often not made until the time of surgery. The definitive diagnosis relies on clinical suspicion and histopathological confirmation. In the treatment of head and neck schwannomas, complete surgical excision with appropriate approaches is efficient. It is important bearing in mind possible vagal or sympathetic chain injury. Local recurrence is extremely rare.

\section{Declaration of patient consent}

The authors certify that they have obtained all appropriate patient consent forms. In the form the patient(s) has/have given his/her/their consent for his/her/their images and other clinical information to be reported in the journal. The patients understand that their names and initials will not be published and due efforts will be made to conceal their identity, but anonymity cannot be guaranteed.

Financial support and sponsorship

Nil.

\section{Conflicts of interest}

There are no conflicts of interest.

\section{References}

1. Chang SC, Schi YM. Neurilemmoma of the vagus nerve: A case report and brief literature review. Laryngoscope 1984;94:946-9.

2. Colreavy MP, Lacy PD, Hughes J, Bouchier-Hayes D, Brennan P, O'Dwyer AJ, et al. Head and neck schwannomas - a 10-year review. J Laryngol Otol 2000;114:119-24.

3. Valentino J, Boggess MA, Ellis JL, Hester TO, Jones RO. Expected neurologic outcome for surgical treatment of cervical neurilemmomas. Laryngoscope 1998;108:1009-13.

4. Russel D, Rubinstein L. Pathology of Tumours of the Nervous System. Baltimore: Williams and Wilkins, 1989.

5. Saito DM, Glastonbury CM, El-Sayed I, Eisele DW. Para- 
pharyngeal space schwannomas. Preoperative imaging determination of the nerve of origin. Arch Otolaryngol Head Neck Surg 2007;133:662-7.

6. Ford LC, Cruz RM, Rumore GJ, Klein J. Cervical cystic schwannoma of vagus nerve: diagnostic and surgical challenge. J Otolaryngol 2003;32:61-3.
7. St Pierre S, Theriault R, Leclerc JE. Schwannomas of the vagus nerve in the head and neck. J Otolaryngol 1985;14:167-70.

8. Fujino $\mathrm{K}$, Shinohara $\mathrm{K}$, Aoki $\mathrm{M}$, Hashimoto $\mathrm{K}$, Omori $\mathrm{K}$. Intracapsular enucleation of vagus nerve-originated tumours for preservation of neural function. Otolaryngol Head Neck Surg 2000;123:334-6. 